TRANSACTIONS OF THE

AMERICAN MATHEMATICAL SOCIETY

Volume 187, Issue 1, 1974

\title{
JORDAN ALGEBRAS AND CONNECTIONS ON HOMOGENEOUS SPACES
}

BY

\author{
ARTHUR A. SAGLE( $\left.{ }^{1}\right)$
}

\begin{abstract}
We use the correspondence between $G$-invariant connections on a reductive homogeneous space $G / H$ and certain nonassociative algebras to explicitly compute the pseudo-Riemannian connections in terms of a Jordan algebra $J$ of endomorphisms. It is shown that if $G$ and $H$ are semisimple Lie groups, then $J$ is a semisimple Jordan algebra. Also a general method for computing examples of $J$ is given.
\end{abstract}

1. Introduction. In this paper we present many algebraic results that are related to the differential geometry of homogeneous spaces and we now give some background results. Thus, let $G$ be a connected Lie group with Lie algebra $g$ and $H$ a closed (Lie) subgroup with Lie subalgebra $b$. The pair $(G, H)$ on $(g, b)$ is called a reductive pair if there exists a subspace $m$ of $g$ such that $g=m+b$ (direct sum) with (Ad $H) m \subset m$; that is, in terms of the algebras $[b, m] \subset m$. The corresponding homogeneous space $G / H$ is called a reductive homogeneous space. In this case if we also have $[m, m] \subset b$, then $(g, b)$ is a symmetric pair and $G / H$ is a symmetric space. We shall be most interested in the case when $(g, b)$ is not a symmetric pair.

In [7] there was established a correspondence between $G$-invariant connections $\nabla$ on a reductive space $G / H$ and certain nonassociative algebras $(m, \alpha)$ where $\alpha$ is a bilinear multiplication function on $m$ as follows.

Theorem. Let $G / H$ be a reductive bomogeneous space with fixed Lie algebra decomposition $g=m+b$ with (Ad $H) m \subset m$. Then there is a one-to-one correspondence between the set of all G-invariant connections $\nabla$ on $G / H$ and the set of nonassociative algebras $(m, a)$ so that $\mathrm{Ad} H$ is contained in the automorphism group of $(m, \alpha)$; that is, $(\operatorname{Ad} P) \alpha(X, Y)=\alpha((\operatorname{Ad} P) X,(\operatorname{Ad} P) Y)$ for all $P \in H$ and $X, Y \in m$.

Received by the editors March 19, 1973.

AMS (MOS) subject classifications (1970). Primary 53C05, 17C50, 17B20; Secondary $53 \mathrm{C} 30,17 \mathrm{~A} 30$.

Key words and phrases. Reductive pair, homogeneous space, pseudo-Riemannian connection, nonassociative algebra, Jordan algebra, Taylor series.

(1) Sponsored in part by NSF Grant GP-29224.

Copyright $\odot 1974$, American Mathematical Society 
If the $G$-invariant connection $\nabla$ on $G / H$ is a pseudo-Riemannian connection that is induced by a nondegenerate form $C$ on $m$, then the algebra satisfies the following conditions; see [4], [7]. The mappings $a(X): m \rightarrow m: Y \rightarrow a(X, Y)$ are $C$-skew symmetric for all $X \in m$ and the mappings Ad $P: m \rightarrow m: Y \rightarrow$ (Ad $P) Y$ satisfy $C((\operatorname{Ad} P) X,(\operatorname{Ad} P) Y)=C(X, Y)$ for all $X, Y \in m$ and $P \in H$. Thus by "differentating" the automorphism and isometry conditions for Ad $H$ we investigate this situation in terms of nonassociative algebras $(m, a)$ which are determined as follows. Let $(g, b)$ be a reductive pair with a fixed direct sum decomposition $g=m+b$ and $[h, m] \subset m$. Let $\alpha: m \times m \rightarrow m$ be a bilinear multiplication function which defines an algebra $(m, \alpha)$ such that ad $U: m \rightarrow m: Y \rightarrow[U, Y]$ for all $U \in b$ is in the derivation algebra of $(m, \alpha)$. Let $C$ be a nondegenerate symmetric bilinear form on $m$ such that the mappings $a(X)$ and ad $U$ for all $X \in m, U \in b$ are $C$-skew symmetric. We now denote such an algebra $(m, a)$ by $(m, a, C)$ and note that in terms of algebras that $(m, a, C)$ determines the corresponding pseudo-Riemannian $G$-invariant connections.

$A$ well behaved example of this situation is when a pseudo-Riemannian connection $\nabla$ is of the first kind; that is, for all $X \in m$ the maps $t \rightarrow \pi \exp t X$ are geodesics where $\pi: G \rightarrow G / H$ is the canonical projection. In this case $a(X, Y)=1 / 2 X Y$ where $X Y=[X, Y]_{m}$ is the projection of $[X, Y]$ in $g$ into the $m$ component of the decomposition $g=m+b$; see [7]. For this case we change the notation and let $B$ denote the corresponding nondegenerate form and let $(m, 1 / 2 X Y, B)$ denote the corresponding algebra. The case where $g$ and $b$ are semisimple gives a reductive pair $(g, b)$ where $g=m+b$ with $m=b^{\perp}$ relative to the Killing form of $g$ and $B$ is the Killing form restricted to $m \times m$; see Corollary $2, \S 2$.

Jordan algebras enter into the analysis of the connection $\nabla$ given by $(m, a, C)$ by comparing $\nabla$ with a given connection of the first kind determined by $(m, 1 / 2 X Y, B)$ as follows. Thus assuming a pseudo-Riemannian connection exists (which we do throughout this paper), we have since $B$ and $C$ are nondegenerate forms that there exists a unique $S \in G L(m)$ such that

$$
C(X, Y)=B(S X, Y) \text {. }
$$

Since $B$ is a symmetric form, $S$ satisfies

$$
S^{b}=S
$$

where $b$ denotes the adjoint relative to $B$. Since ad $U$ is $B$ - and $C$-skew symmetric, $S$ also satisfies

$$
[\operatorname{ad} U, S]=0
$$


for all $U \in b$. For $B(S$ ad $U X, Y)=C(\operatorname{ad} U X, Y)=-C(X$, ad $U Y)=$ - $B(S X$, ad $U Y)=B($ ad $U S X, Y)$.

The set of endomorphisms of $m$ satisfying (2) and (3) form a Jordan algebra $J$ relative to the usual operation $S_{1} S_{2}=1 / 2\left(S_{1} \circ S_{2}+S_{2} \circ S_{1}\right)$ where $\circ$ is compos tion of endomorphisms. Thus the connection $\nabla$ determined by $(m, \alpha, C)$ corresponds to a unique invertible element $S$ in J. Now from results in [4], [9] the formula for $\alpha$ is given by

$$
2 \alpha(X, Y)=X Y+S^{-1}[X(S Y)-(S X) Y]
$$

where $1 / 2 X Y$ is the multiplication in $(m, 1 / 2 X Y, B)$. Thus given an invertible element in $J$ we can define the connection $\nabla$ corresponding to $(m, \alpha, C)$ by using forinulas (1) and (4) to determine $C$ and $a$. However for any $T \in J, S=\exp T$ is invertible in $J$. Thus in this way there is a correspondence between the set of connections (determined above by $(m, a, C)$ ) and the elements of $J$. Furthermore, using (4) these connections are parameterized by elements of the symmetric space $M=J \cap G L(m)$; see [5]. Note that for $S=I \in J \cap G L(m)$ we obtain the original algebra $(m, 1 / 2 X Y, B)$ so that $M$ is nonempty.

We now note that the relationship between Jordan algebras and connections is rather general. For in the case of a Lie group $G$ (with $H=\{e\}$ ) if we assume the algebra $(g, 1 / 2[X, Y], B)$ exists where ad $X$ is $B$-skew symmetric, then the corresponding Jordan algebra is $J=\left\{S \in \operatorname{End}(g): S^{b}=S\right\}$. Conversely, given any Jordan algebra $J$ of endomorphisms of a vector space $V$ which are symmetric relative to a nondegenerate form $B$ on $V$, then we can regard $V$ as an abelian Lie algebra and form $(g, 1 / 2[X, Y], B)$. Thus the corresponding Jordan algebra of this triple is just $J$.

In the remaining sections we discuss mainly reductive pairs $(g, b)$ with $g$ and $b$ semisimple. We compare $(m, a, C)$ with $(m, 1 / 2 X Y, B)$ where $B$ is the Killing form restricted to $m \times m$ as mentioned above. Thus we find a direct sum decomposition $m=K_{0}+K_{1}+K_{2}$ into ad $b$-invariant subspaces and show $J$ is semisimple. We give examples of this decomposition and $J$, but leave the complete classification of $J$ as an open problem. This is a hard problem taking into account the classification of semisimple subalgebras of a semisimple Lie algebra [1]. Finally in $\$ 5$ we give some sample applications involving the connection and curvature of $G / H$ regarded as functions of $M=J \cap G L(m)$.

2. Semisimplicity of $J$. Let $(g, b)$ be a reductive pair with decomposition $g=m+b$ and $(\operatorname{ad} b) m \subset m$. From the Lie algebra identities for $g$, it is easy to see that ad $b \subset \operatorname{Der}(m, 1 / 2 X Y)$ which is the derivation algebra of $(m, 1 / 2 X Y)$. We now assume there is a symmetric nondegenerate form $B$ on $m$ so that $(m, 1 / 2 X Y, B)$ 
exists; that is, for all $X \in m, U \in b$ the mappings

$$
L(X): m \rightarrow m: Y \rightarrow X Y \text { and ad } U: m \rightarrow m: Y \rightarrow[U, Y]
$$

are $B$-skew symmetric. Now let $(m, a, C)$ be another algebra as discussed in $\S 1$; specifically, $(m, \alpha)$ is an algebra and $C$ is a symmetric nondegenerate form so that for all $X \in m, U \in b$ the mappings $a(X): m \rightarrow m: Y \rightarrow a(X, Y)$ and ad $U: m \rightarrow m: Y \rightarrow[U, Y]$ are $C$-skew symmetric where ad $b \subset \operatorname{Der}(m, a)$ which is the derivation algebra of $(m, a)$. Let $J=\{S \in \operatorname{End}(m):[S$, ad $U]=0$ all $U \in b$ and $\left.S^{b}=S\right\}$ be the Jordan algebra which relates all the possible algebras $(m, a, C)$ to $(m, 1 / 2 X Y, B)$ as previously discussed. We now have the following.

Theorem 1. Let $(g, b)$ and $(m, 1 / 2 X Y, B)$ be as above and let ad $b$ be completely reducible in $m$. Then $J$ is a semisimple Jordan algebra.

Proof. Let $A$ be the associative subalgebra of $\operatorname{End}(m)$ given by

$$
A=\{T \in \operatorname{End}(m):[T, \text { ad } U]=0 \text { all } U \in b\} .
$$

Then $A$ is closed under the involution $b$ induced by $B$; that is, $B(T X, Y)=$ $B\left(X, T^{b} Y\right)$. For, from $0=[T, \operatorname{ad} U]$ we see $0=\left[T^{b}\right.$, ad $\left.U\right]$ using $(\operatorname{ad} U)^{b}=$ - ad $U$. Thus $A$ is an associative algebra with involution and the set $H(A, b)=$ $\left\{T \in A: T^{b}=T\right\}$ of $B$-symmetric elements is a Jordan algebra relative to the usual multiplication. From [6] we see that the Jordan radical is given by $\operatorname{Rad} H(A, b)=H(A, b) \cap \operatorname{Rad} A$ where $\operatorname{Rad} A$ is the radical of the associative algebra $A$. Therefore noting that $J=H(A, b)$ it suffices to show $A$ is semisimple.

Thus since ad $b$ is completely reducible on $m$, we see from [3, p. 102] that the centralizer

$$
C=\{T \in g l(m):[T, \text { ad } U]=0 \text { all } U \in b\}
$$

is completely reducible on $m$. But identifying $g l(m)$ with $\operatorname{End}(m)$, this means $A$ is completely reducible on $m$. This implies $A$ is semisimple.

Corollary 2. Let $b$ be a semisimple subalgebra of the semisimple Lie algebra $g$. Then $(g, b)$ is a reductive pair with decomposition $g=m+b$ where $m=b^{\perp}$ relative to the Killing form, Kill, of $g$. Also for $X, Y \in m$, the form $B(X, Y)=\operatorname{Kill}(X, Y)$ is nondegenerate on $m$ and the algebra $(m, 1 / 2 X Y, B)$ satisfies $L(X)$ and ad $U$ are $B$-skew symmetric for all $X \in m, U \in b$. In this case the Jordan algebra $J$ is semisimple.

Proof. The last statement follows by noting ad $b$ is semisimple and therefore is completely reducible in $m$. The first parts were proven in [10] which we now sketch. Since $b$ is semisimple, the Killing form of $g$ restricted to $b$ is 
nondegenerate. Thus we can decompose $g=m+b$ with $m=b^{\perp}$. With $B$ as above we see ad $U$ is $B$-skew symmetric for $U \in b$ and the map $L(X): m \rightarrow m: Y \rightarrow X Y$ is $B$-skew symmetric as follows. Let $b(X, Y)=[X, Y]$ be the projection of $[X, Y]$ in $g$ into $b$, then since $m=b^{\perp}$ we have $B(L(X) Y, Z)=B(X Y+b(X, Y), Z)=$ $\operatorname{Kill}([X, Y], Z)=-\operatorname{Kill}(Y,[X, Z])=-B(Y, X Z+b(X, Z))=-B(Y, L(X) Z)$.

In the next sections we obtain a more detailed decomposition for $m$ and $J$ in the case $g$ is semisimple and $b$ is semisimple.

3. Decompositions for $m$ and $J$. We continue the discussion of the previous section and obtain a decomposition for $m$ (Theorem 3). Some of these results are buried in [9] and we repeat them for convenience. For any reductive pair $(g, b)$ with decomposition $g=m+b$ and $(\operatorname{ad} b) m \subset m$, the identities of $g$ induce identities on $(m, X Y)$ as follows. As before for $X, Y \in m$ let $[X, Y]=X Y+$ $b(X, Y)$ where $X Y=[X, Y]_{m}$ is the projection of $[X, Y]$ into $m$ and similarly $b(X, Y)=[X, Y]_{b}$. For $U \in b$ we also use the notation $D(U)=$ ad $U$ restricted to $m$ and $D(X, Y)=D(b(X, Y))$. Then we have

$$
\begin{aligned}
& X Y=-Y X, \text { bilinear } \\
& D(X, Y)=-D(Y, X), \text { bilinear } \\
& D(X, Y) Z+D(Y, Z) X+D(Z, X) Y=X(Y Z)+Y(Z X)+Z(X Y) \\
& D(X Y, Z)+D(Y Z, X)+D(Z X, Y)=0 \\
& {[D(U), D(X, Y)]=D(D(U) X, Y)+D(X, D(U) Y)} \\
& D(U)(X Y)=(D(U) X) Y+X(D(U) Y) .
\end{aligned}
$$

Now assume $g$ and $b$ are semisimple; then from Corollary 2 we see that $g=m+b$ with $m=b^{\perp}$ relative to the Killing form on $g$ and that $B(X, Y)=\operatorname{Kill}(X, Y)$ is nondegenerate and $L(X)$ and $D(U)$ are $B$-skew symmetric. Next let

$$
K_{0}=\{X \in m:(\operatorname{ad} U) X=0 \text { for all } U \in b\}
$$

then using (3.6) and (3.3) we see that $K_{0}$ is a Lie subalgebra of $(m, X Y)$. We also have

$$
b\left(K_{0}, m\right)=0
$$

for if $W \in K_{0}, Z \in m$ and $U \in b$, then

$$
\begin{aligned}
0 & =\operatorname{Kill}_{\boldsymbol{g}}((\operatorname{ad} U) W, Z)=\operatorname{Kill}_{\boldsymbol{g}}([U, W], Z)=\operatorname{Kill}_{\boldsymbol{g}}(U,[W, Z]) \\
& =\operatorname{Kill}_{\boldsymbol{g}}(U, W Z+b(W, Z))=\operatorname{Kill}_{\boldsymbol{g}}(U, b(W, Z)) \text {, using } m=b^{\perp}
\end{aligned}
$$


and since $\mathrm{Kill} \mid b \times b$ is nondegenerate, $b(W, Z)=0$. From this we see $\left[K_{0}, m\right]=$ $K_{0} m \subset m$ so that $m$ is a ad $K_{0}$-module and also $L: K_{0} \rightarrow L\left(K_{0}\right)$ is a representation of $K_{0}$ on $m$ where $L\left(K_{0}\right)=\left\{L(V): V \in K_{0}\right\}$. Thus let $V, W \in K_{0}$ on $Z \in m$, then from (3.3) and (3.7) we obtain $0=V(W Z)+W(Z V)+Z(V W)=([L(V), L(W)]-$ $L(V W)) Z$ which gives the result

$$
L(V W)=[L(V), L(W)]
$$

A similar computation shows that $L\left(K_{0}\right) \subset \operatorname{Der}(m, X Y)$. Also since $D(U)$ is a derivation of $(m, X Y)$, noting (3.6), we have $[D(U), L(V)]=L(D(U) V)=0$ for $V \in K_{0}$ and $U \in b$.

Next we use some results on centralizers to show $K_{0}=c \oplus K_{0}^{\prime}$ where $c$ is the center of $K_{0}$ and $K_{0}^{\prime}$ is zero or a semisimple Lie algebra in ( $m, X Y$ ). Since $g$ and $b$ are semisimple, $L=$ ad $g$ and $L_{1}=$ ad $b$ are completely reducible on $g$. Therefore the centralizer, $C_{L}\left(L_{1}\right)$, of $L_{1}$ in $L$ is completely reducible on $g$ [3, p. 102]. But

$$
C_{L}\left(L_{1}\right)=\{\operatorname{ad} W \in \operatorname{ad} g:[\operatorname{ad} b, \operatorname{ad} W]=0\}=\{\operatorname{ad} W \in \operatorname{ad} g: \operatorname{ad}[b, W]=0\} .
$$

Since $g$ is seinisimple, $\operatorname{ad}[b, W]=0$ implies $[b, W]=0$. We now show $W \in K_{0}$ as follows. Let $W=W_{1}+W_{2}$ where $W_{1} \in m$ and $W_{2} \in b$, then for $U \in b, 0=[U, W]=$ $\left[U, W_{1}\right]+\left[U, W_{2}\right]$. From the ad $b$-invariant direct sum $g=m+b$, this implies $\left[b, W_{i}\right]=0$. But since $b$ is semisimple and $\left[b, W_{2}\right]=0$ we see $W_{2}=0$; thus $W=$ $W_{1} \in m$ which means $W_{1} \in K_{0}$. This implies $C_{L}\left(L_{1}\right)=\operatorname{ad}_{g} K_{0}$ is completely reducible in $g$ and therefore completely reducible on ad ${ }_{8} K_{0}$-submodules of $g$.

Next for $X \in m$ and $V \in K_{0}$ we see

$$
\operatorname{ad}_{\boldsymbol{g}} V(X)=[V, X]=V X+b(V, X)=L(V) X \text {, using (3.7) }
$$

so that the action of $\operatorname{ad}_{8} K_{0}$ and $L\left(K_{0}\right)$ on $m$ are the same. Since $m$ is an ad $K_{8} K_{0^{-}}$ submodule, this inplies $L\left(K_{0}\right)$ is completely reducible on $m$. Thus from [3] we may write $L\left(K_{0}\right)=L(c) \oplus L\left(K_{0}^{\prime}\right)$ for suitable subsets $c$ and $K_{0}^{\prime}$ of $K_{0}$ and where $L(c)$ is the center of $L\left(K_{0}\right)$ and $L\left(K_{0}^{\prime}\right)$ is zero or semisimple. Now the map $K_{0} \rightarrow$ $L\left(K_{0}\right): V \rightarrow L(V)$ is an isomorphism of Lie algebras. For it is a homomorphism using (3.8) and $L(V)=0$ implies ad ${ }_{g} V=0$ so that $V=0$. From this isomorphism we obtain the desired result

$$
K_{0}=c \oplus K_{0}^{\prime} .
$$

Next we consider a direct sum decomposition for $m$ and show $B \mid K_{0} \times K_{0}$ is nondegenerate (recall $B(X, Y)=\operatorname{Kill}(X, Y)$ ). Since ad $b$ is completely reducible on $m$ and $K_{0}$ is ad $b$-invariant we have a direct sum $m=K_{0}+b$ where $b$ is 
ad $b$-invariant. Furthermore $(\operatorname{ad} b) b=b$. For if $p=(\operatorname{ad} b) b$ is a proper ad $b$ subinodule of $b$, then by the complete reducibility of ad $b$ on $b$, we can find an ad $b$-invariant coinplement $p^{\prime}$ with $b=p^{\prime}+p$ (direct sum). Thus (ad $\left.b\right) p^{\prime} \subset p^{\prime} \cap$ (ad $b) b=p^{\prime} \cap p=0$ so that $p^{\prime} \subset K_{0}$, a contradiction. Thus $m=K_{0}+b$ with $(\operatorname{ad} b) b=b$.

To see that $B \mid K_{0} \times K_{0}$ is nondegenerate, let $U \in K_{0}$ be such that $B\left(U, K_{0}\right)=0$. Then

$$
B(U, m)=B\left(U, K_{0}+b\right)=B(U,(\operatorname{ad} b) b)=-B((\operatorname{ad} b) U, b)=0
$$

and since $B \mid m \times m$ is nondegenerate, $U=0$. We also have $B \mid c \times c$ and $B \mid K_{0}^{\prime} \times K_{0}^{\prime}$ are nondegenerate.

Since $B \mid K_{0} \times K_{0}$ is nondegenerate we can decompose $m=K_{0}+b$ where we can assume $b=K_{0}^{1}$ relative to $B$, ( $\left.\operatorname{ad} b\right) b=b$ and $B \mid b \times b$ is nondegenerate. Using $b=K_{0}^{\perp}$ note that $L\left(K_{0}\right) b C b$ since $B\left(K_{0}, K_{0} b\right)=B\left(K_{0} K_{0}, b\right)=0$ using $K_{0}$ is a subalgebra of $(m, X Y)$. Now let

$$
K_{1}=\left\{Y \in b: L(U) Y=0 \text { all } U \in K_{0}\right\}
$$

then since $L\left(K_{0}\right) \subset \operatorname{Der}(m, X Y)$ (remarks following (3.8)), we see $K_{1}$ is a subalgebra of $(m, X Y)$. Also from the remarks following (3.8) we see $[L(U)$, ad $P]=$ 0 for $U \in K_{0}, P \in b$ so that $K_{1}$ is ad $b$-invariant.

Since $L\left(K_{0}\right)$ is completely reducible in $m$ and $b$ is an $L\left(K_{0}\right)$-submodule, we can decompose $b=K_{1}+K_{2}$ into $L\left(K_{0}\right)$-submodules. In a manner similar to the proof that $B \mid K_{0} \times K_{0}$ is nondegenerate, we see $B \mid K_{1} \times K_{1}$ is nondegenerate. Thus we can assume $K_{2}=K_{1}^{\perp}$ relative to $B \mid b \times b$ and we have that $K_{2}$ is $L\left(K_{0}\right)$ - and ad $b$-invariant and $B \mid K_{2} \times K_{2}$ is nondegenerate. We summarize some of these results.

Theorem 3. (a) Let $g$ be a semisimple Lie algebra and $b$ a semisimple subalgebra, then there is a decomposition $g=m+b$ where $m=b^{\perp}$ relative to the Killing form of $g$ and $(g, b)$ is a reductive pair.

(b) The algebra $(m, X Y)$ is sucb that $m=c+K_{0}^{\prime}+K_{1}+K_{2}$ as an ad $b$ invariant direct sum and satisfies the multiplicative relations

$$
\begin{aligned}
& c c=c K_{0}^{\prime}=c K_{1}=0, \quad c K_{2} \subset K_{2}, \\
& K_{0}^{\prime} K_{0}^{\prime}=K_{0}^{\prime}, \quad K_{0}^{\prime} K_{1}=0, \quad K_{0}^{\prime} K_{2} \subset K_{2}, \\
& K_{1} K_{1} \subset K_{1}, \quad K_{1} K_{2} \subset K_{2}, \quad K_{2} K_{2} \subset m_{m}
\end{aligned}
$$

(c) $K_{0}=c+K_{0}^{\prime}$ is such that $(\operatorname{ad} b) K_{0}=0$ and $K_{0}^{\prime}$ is zero or a semisimple Lie subalgebra of $(m, X Y)$. Also $(\operatorname{ad} b) K_{i}=K_{i}$ for $i=1,2$. 
(d) $b+K_{0}$ is a Lie subalgebra of $g$ and $m$ is an $\operatorname{ad}\left(b+K_{0}\right)$-module. $L\left(K_{0}\right) \subset$ $\operatorname{Der}(m, X Y)$ and $\left[L\left(K_{0}\right)\right.$, ad $\left.b\right]=0$. Also $L\left(K_{0}\right) K_{2}=K_{2}$ and $K_{2}$ is $\operatorname{ad}\left(b+K_{0}+K_{1}\right)$-invariant.

Proof. We have previously discussed (a) and most of (b) except $K_{1} K_{2} \subset K_{2}$. To see this we let $U \in K_{1}, V \in K_{2}$ and $U V=X+Y+Z$ where $X \in K_{0}, Y \in K_{1}$ and $Z \in K_{2}$. Now $X=0$ as follows. For any $P \in K_{0}$,

$$
\begin{aligned}
B(P, X) & =B(P, U V-Y-Z)=B(P, U V), \text { orthogonality } \\
& =B(P U, V)=0
\end{aligned}
$$

using $K_{0} K_{1}=0$. Thus since $B \mid K_{0} \times K_{0}$ is nondegenerate, $X=0$. Similarly let $Q \in K_{1}$, then

$$
B(Q, Y)=B(Q, U V)=B(Q U, V)=0
$$

using $Q U \in K_{1} K_{1} \subset K_{1}$ and $B\left(K_{1}, K_{2}\right)=0$. Since $B \mid K_{2} \times K_{2}$ is nondegenerate, $Y=0$.

Parts (c) and (d) have been discussed except showing $K_{2}$ is $\operatorname{ad}\left(b+K_{0}+K_{1}\right)$-invariant. To see this we note that $\operatorname{ad}\left(b+K_{0}\right) K_{2}=(\operatorname{ad} b) K_{2}+$ ( $\left.\operatorname{ad} K_{0}\right) K_{2} \subset K_{2}$, recalling (ad $\left.K_{0}\right) K_{2}=L\left(K_{0}\right) K_{2} \subset K_{2}$. Thus we must show (ad $\left.K_{1}\right) K_{2}=K_{1} K_{2}+b\left(K_{1}, K_{2}\right)$ is in $K_{2}$ which follows from (b) and $b\left(K_{1}, K_{2}\right)=$ 0 . To see this note

$$
\begin{aligned}
0 & =\operatorname{Kill}\left(\left[b, K_{1}\right], K_{2}\right), \text { using } K_{2}=K_{1}^{\perp} \\
& =\operatorname{Kill}\left(b,\left[K_{1}, K_{2}\right]\right) \\
& =\operatorname{Kill}\left(b, b\left(K_{1}, K_{2}\right)\right), \text { using } m=b^{\perp} .
\end{aligned}
$$

Thus since Kill $\mid b \times b$ is nondegenerate, $b\left(K_{1}, K_{2}\right)=0$.

We shall give some examples later, but it should be noted that the problem of finding "holonomy irreducible" connections determined by $(m, a, C)$ leads to the problem of finding algebras $(m, a, C)$ which have no left ideals which are ad $b$-invariant. In particular these algebras must be simple; see [9]. The simplest examples of this type were discussed in [9, Proposition 3] where $S \mid K_{i}=\lambda_{i} I$ and relations between the $\lambda_{i}$ 's were determined. A general analysis of the algebras $(m, a, C)$ probably must follow the lines of Lie algebras or extended Lie algebras [8] since identity elements or idempotents need not be in $(m, a, C)$. Thus using $C(\alpha(X, Y), Z)=-C(Y, a(X, Z))$ we see that if $C$ is nondegenerate and $E$ is an identity element, then

$$
C(X, Y)=C(\alpha(E, X), Y)=-C(X, \alpha(E, Y))=-C(X, Y)
$$


a contradiction. If $C$ is positive definite and $E$ is an idempotent we obtain

$$
C(E, E)=C(\alpha(E, E), E)=-C(E, \alpha(E, E))=-C(E, E),
$$

a contradiction.

Proposition 4. Let $g$ be a semisimple Lie algebra with $b$ a semisimple subalgebra so that $(g, b)$ is a reductive pair with $g=m+b$ where $m=b^{\perp}$. If $m=K_{0}+b$ is the decomposition as before, then $J K_{0}=K_{0}$ and $J b=b$. Thus $J=J_{0} \oplus J(b)$ is the direct sum of ideals where $J_{0}=J \mid K_{0}$ and $J(b)=J \mid b$. Furtbermore $J_{0}$ is isomorphic to the Jordan algebra of $r \times r$ B-symmetric matrices where $r$ is the dimension of $K_{0}$.

Proof. Let $U \in K_{0}$ and $S \in J$, then since $[\operatorname{ad} b, S]=0$ we have ad $b(S U)=$ $S($ ad $b U)=0$ so that by definition $S K_{0} \subset K_{0}$. Since $I \in J$, we have $J K_{0}=K_{0}$. Since $b=K_{0}^{\perp}$ and $S$ is $B$-symmetric we also have $J b=b$.

Next relative to $m=K_{0}+b$ we see that ad $b \mid m$ and $S \in J$ have the matrices of the form

$$
\left[\begin{array}{ll}
0 & \\
& b_{22}
\end{array}\right] \text { and }\left[\begin{array}{ll}
S_{11} & 0 \\
0 & S_{22}
\end{array}\right] \text {. }
$$

Thus the condition $[\mathrm{ad} h, S]=0$ gives no restriction on $S_{11}$. Therefore the set of matrices $S_{11}$ which is isomorphic to $J_{0}$ has the only restrictions $S_{11}^{b}=S_{11}$. That is, $J_{0}$ is isomorphic to $\left\{T \in \operatorname{End}\left(K_{0}\right): T^{b}=T\right\}$ where $b_{0}$ is the adjoint relative to the nondegenerate form $B_{0}=B \mid K_{0} \times K_{0}$.

Examples in the next section lead to the following.

Conjecture. Let $m=K_{0}+K_{1}+K_{2}$ as before with $K_{0} \neq 0$, then $J K_{i}=K_{i}$ for $i=0,1,2$.

If this is the case, then $J=J_{0} \oplus J_{1} \oplus J_{2}$ where $J_{i}=J \mid K_{i}$ are ideals in $J$.

4. Examples. We now consider the decomposition of the previous sections for various simple matrix algebras $g$ where the semisimple subalgebra $b$ is imbedded in $g$ in a natural way.

To see easily that $J K_{i}=K_{i}$ we recall some facts on homogeneous components for an associative algebra [2, p. 124]. The associative algebra $A$ we want to consider is the algebra generated by ad $b \mid \mathrm{m}$. Since we are assuming $b$ is semisimple, it is completely reducible; thus $A$ is completely reducible and semisimple. Furthermore $A K_{0}=0$ and $A b=b$ so that we shall restrict our attention to $A \mid b \equiv \bar{A}$.

Now let $\Gamma=\{T \in \operatorname{End}(b):[T, P]=0$ for all $P \in \bar{A}\}$ be the centralizer of $\bar{A}$. 
Then we see $J(b) \subset \Gamma$ and $J(b)$ consists of the $B_{1}=B \mid b \times b$ symmetric elements. Let $n$ be an $\bar{A}$-irreducible subinodule of $b$, then the homogeneous component $p$ determined by $n$ is $p=\Sigma n_{i}$ summed over all $\bar{A}$-irreducible submodules $n_{i}$ which are $\bar{A}$-isomorphic to $n$. In this case we have $\Gamma p=p$ and $p$ is a $\Gamma$-completely reducible submodule of $b$. In the examples we shall show that the $\bar{A}$-homogeneous components of $b=K_{1}+K_{2}$ are either in $K_{1}$ or $K_{2}$; thus $\Gamma K_{1}=K_{1}$ and $\Gamma K_{2}=K_{2}$.

The general idea behind the examples is as follows. Let $g$ be represented by matrices and suppose $b=b_{1} \oplus b_{2} \oplus \cdots \oplus b_{t}$ as a direct sum of simple ideals; consider the case $b=b_{1} \oplus b_{2}$. In this case represent $g$ by matrices

$$
\left[\begin{array}{lll}
A_{11} & A_{12} & A_{13} \\
A_{21} & A_{22} & A_{23} \\
A_{31} & A_{32} & A_{33}
\end{array}\right]
$$

and imbed $b$ as matrices of the form

$$
\left[\begin{array}{lll}
0 & & 0 \\
& b_{22} & \\
0 & & b_{33}
\end{array}\right] \text {. }
$$

There is a 0 in the $(1,1)$ position because $K_{0}$ is not zero unless $A_{11}=0$. From matrix computations we roughly obtain

$$
\begin{aligned}
& K_{0} \text { is the set of matrices }\left[\begin{array}{lll}
A_{11} & 0 & 0 \\
0 & 0 & 0 \\
0 & 0 & 0
\end{array}\right] ; \\
& K_{1} \text { is the set of matrices }\left[\begin{array}{llr}
0 & 0 & 0 \\
0 & 0 & A_{23} \\
0 & A_{32} & 0
\end{array}\right] ; \\
& K_{2} \text { is the set of matrices }\left[\begin{array}{lrr}
0 & A_{12} & A_{13} \\
A_{21} & 0 & 0 \\
A_{31} & 0 & 0
\end{array}\right] .
\end{aligned}
$$

Example. (1) Let $g=s o(n)$ be the $n \times n$ skew-symmetric matrices and $b=$ so( $k$ ) the $k \times k$ skew-symmetric matrices where $k<n-1$. As above regard $g$ as matrices 


$$
\left[\begin{array}{ll}
A_{11} & A_{12} \\
A_{21} & A_{22}
\end{array}\right]
$$

where $A_{i i}$ are skew-symmetric and imbed $b$ as matrices of the form

$$
\left[\begin{array}{ll}
0 & 0 \\
0 & A_{22}
\end{array}\right] \text {. }
$$

Then a straightforward computation, using $\operatorname{Kill}(P, Q)=\lambda \operatorname{tr} P Q$ for $P, Q \in g$, shows $m=b^{\perp}$ is of the form

$$
\left[\begin{array}{lr}
A_{11} & A_{12} \\
A_{21} & 0
\end{array}\right] .
$$

From this we see $K_{0}$ are matrices of the form

$$
\left[\begin{array}{ll}
A_{11} & 0 \\
0 & 0
\end{array}\right]
$$

and $b=K_{0}^{\perp}$ are matrices of the form

$$
\left[\begin{array}{lr}
0 & A_{12} \\
A_{21} & 0
\end{array}\right] .
$$

Now for $U \in K_{0}, V \in b$ we compute $L(U) V=[U, V]=0$ to obtain

$$
A_{11} A_{12}=A_{21} A_{11}=0
$$

Let $r=n-k$, then since $K_{0}$ consists of all $r \times r$ skew-symmetric matrices we see that (*) implies $A_{21}=0$; that is, $K_{1}=0$ and $b=K_{2}$. Thus $m=K_{0}+K_{2}$ with the above matrix description.

Now we see that $J=J_{0} \oplus J_{2}$ as given at the end of $\S 3$. In this case $J_{0}$ is the simple Jordan algebra of symmetric $r \times r$ matrices. We now consider $J_{2}$.

Since $A_{12}^{t}=-A_{21}$ where ${ }^{t}$ is the transpose, we set $A=A_{12}$, then

$$
\left[\begin{array}{ll}
0 & A \\
-A^{t} & 0
\end{array}\right] \in K_{2}
$$

and we let $A_{i}$ be a $r \times k$ matrix of the form 


$$
A_{i}=\left[\begin{array}{c}
0 \\
a_{i 1} a_{i 2} \cdots a_{i k} \\
0
\end{array}\right]
$$

and let $p_{i}$ be the set of matrices of the form

$$
\left[\begin{array}{ll}
0 & A_{i} \\
-A_{i}^{t} & 0
\end{array}\right]
$$

Then a computation shows ad $b p_{i}=p_{i}$, using $(\mathrm{ad} b)^{t}=-$ ad $b$. Furthermore the $p_{i}$ are ad $b$-irreducible and $K_{2}=p_{1}+\cdots+p_{r}$. We have $p_{i}$ and $p_{j}$ are ad $b$-isomorphic using the obvious map. Thus let $\left(\alpha_{1}, \cdots, a_{k}\right)$ be an arbitrary $k$-tuple in $R^{k}$ and let

$$
\begin{aligned}
& A_{i}=\left[\begin{array}{c}
0 \\
\alpha_{1} \ldots \alpha_{k} \\
0
\end{array}\right] \text { th row, and } \\
& A_{j}=\left[\begin{array}{c}
0 \\
\alpha_{1} \cdots \alpha_{k} \\
0
\end{array}\right] \text { th row. }
\end{aligned}
$$

Then the map $p_{i} \rightarrow p_{j}$ given by

$$
\left[\begin{array}{lr}
0 & A_{i} \\
-A_{i}^{t} & 0
\end{array}\right] \rightarrow\left[\begin{array}{lr}
0 & A_{j} \\
-A_{j}^{t} & 0
\end{array}\right]
$$

is an ad $b$-isomorphism. Thus since $K_{2}=\Sigma p_{i}$ we see $K_{2}$ is a homogeneous component and this is an orthogonal decomposition.

Use the decomposition $K_{2}=\Sigma_{i=1}^{r} p_{i}$ to obtain a basis for $K_{2}$ as follows. Let $E_{1}, \cdots, E_{k}$ be a basis of $p_{1}$ and let ad $b E_{s}=\Sigma_{j} a_{j s} E_{j}$ define a matrix $b_{11}$ for ad $b$ on $p_{1}$ : Let $\phi: p_{1} \rightarrow p_{i}$ be an ad $b$-(or an $\bar{A}$ )-isomorphism and let $E_{s}^{i}=$ $\phi\left(E_{s}\right)$ give a corresponding basis of $p_{i}$. Then ad $b E_{s}^{i}=\operatorname{ad} b \phi\left(E_{s}\right)=\phi\left(\operatorname{ad} b E_{s}\right)=$ $\Sigma_{j} a_{j s}\left(\phi E_{j}\right)=\Sigma_{j} a_{j s} E_{s}^{i}$. This defines a matrix $b_{i i}$ for ad $b$ on $p_{i}$ and $b_{i i}=b_{11}$. Thus relative to a basis of $K_{2}$ chosen this way ad $b \mid K_{2}$ has matrix

$$
\left[\begin{array}{lll}
b_{11} & & 0 \\
& \ddots & \\
0 & & b_{r r}
\end{array}\right]
$$


where $b_{11}=b_{i i}$ for $i=1, \cdots, r$.

Next relative to this basis write the matrix for $S \mid K_{2}$ in block form of $k \times k$ matrices

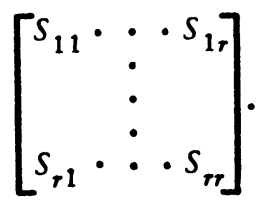

Then $[\mathrm{ad} h, S]=0$ gives the relations

$$
\left[b_{i i}, S_{i i}\right]=b_{i i} S_{i j}-S_{i j} b_{j i}=0 \text {. }
$$

Since $b_{i i}=b_{11}$ we obtain $\left[b_{11}, s_{i j}\right]=0$. But $b_{11}$ represents any $k \times k$ skewsymmetric matrix and this implies $S_{i j}=\lambda_{i j} I$ for $\lambda_{i j} \in R$ and $I$ is the $k \times k$ identity matrix. (If necessary, just compute.) Thus since $S^{t}=S$, the ideal $J_{2}=J \mid K_{2}$ is isomorphic to the $r \times r$ symmetric matrices, $r=n-k$.

Example. (2) We now consider when $b$ is actually semisimple. Thus let $g=s o(n)$ be given by block matrices

$$
\left[\begin{array}{llll}
A_{11} & A_{12} & A_{13} & A_{14} \\
A_{21} & A_{22} & A_{23} & A_{24} \\
A_{31} & A_{32} & A_{33} & A_{34} \\
A_{41} & A_{42} & A_{43} & A_{44}
\end{array}\right]
$$

and let $b=s o\left(k_{1}\right) \oplus s o\left(k_{2}\right) \oplus s o\left(k_{3}\right)$ be imbedded as matrices of the form

$$
\left[\begin{array}{rrrr}
0 & 0 & 0 & 0 \\
0 & A_{22} & 0 & 0 \\
0 & 0 & A_{33} & 0 \\
0 & 0 & 0 & A_{44}
\end{array}\right]
$$

similar to the first example and let $n=r+k_{1}+k_{2}+k_{3}$ where $r>1$. As in the preceding example we have $m$ given by matrices of the form

$$
\left[\begin{array}{cccc}
A_{11} & A_{12} & A_{13} & A_{14} \\
A_{21} & 0 & A_{23} & A_{24} \\
A_{31} & A_{32} & 0 & A_{34} \\
A_{41} & A_{42} & A_{43} & 0
\end{array}\right]
$$


and $m=K_{0}+K_{1}+K_{2}$ where the $K_{i}$ are given by matrices of the form

$$
\begin{aligned}
& K_{0}:\left[\begin{array}{llll}
A_{11} & 0 & 0 & 0 \\
0 & 0 & 0 & 0 \\
0 & 0 & 0 & 0 \\
0 & 0 & 0 & 0
\end{array}\right], \\
& K_{1}:\left[\begin{array}{lllr}
0 & 0 & 0 & 0 \\
0 & 0 & A_{23} & A_{24} \\
0 & A_{32} & 0 & A_{34} \\
0 & A_{42} & A_{43} & 0
\end{array}\right], \\
& K_{2}:\left[\begin{array}{lllr}
0 & A_{12} & A_{13} & A_{14} \\
A_{21} & 0 & 0 & 0 \\
A_{31} & 0 & 0 & 0 \\
A_{41} & 0 & 0 & 0
\end{array}\right] .
\end{aligned}
$$

As in the preceding example we use the skew-symmetry of matrices in $s o(n)$ to obtain the following decomposition $K_{1}=p_{1}+p_{2}+p_{3}$ where the $p_{i}$ are given by

$$
\begin{aligned}
& p_{1}:\left[\begin{array}{rrrr}
0 & 0 & 0 & 0 \\
0 & 0 & A_{23} & 0 \\
0 & A_{32} & 0 & 0 \\
0 & 0 & 0 & 0
\end{array}\right], \\
& p_{2}:\left[\begin{array}{rrrr}
0 & 0 & 0 & 0 \\
0 & 0 & 0 & A_{24} \\
0 & 0 & 0 & 0 \\
0 & A_{42} & 0 & 0
\end{array}\right], \\
& p_{3}:\left[\begin{array}{rrrr}
0 & 0 & 0 & 0 \\
0 & 0 & 0 & 0 \\
0 & 0 & 0 & A_{34} \\
0 & 0 & A_{43} & 0
\end{array}\right] .
\end{aligned}
$$


Next $K_{2}=q_{1}+q_{2}+q_{3}$ where the $q_{i}$ are given by

$$
\begin{aligned}
& q_{1}:\left[\begin{array}{lrrr}
0 & A_{12} & 0 & 0 \\
A_{21} & 0 & 0 & 0 \\
0 & 0 & 0 & 0 \\
0 & 0 & 0 & 0
\end{array}\right], \\
& q_{2}=\left[\begin{array}{lrrr}
0 & 0 & A_{13} & 0 \\
0 & 0 & 0 & 0 \\
A_{31} & 0 & 0 & 0 \\
0 & 0 & 0 & 0
\end{array}\right], \\
& q_{3}:\left[\begin{array}{lllr}
0 & 0 & 0 & A_{14} \\
0 & 0 & 0 & 0 \\
0 & 0 & 0 & 0 \\
A_{41} & 0 & 0 & 0
\end{array}\right] .
\end{aligned}
$$

The homogeneous components of ad $b$ (or $\bar{A}$ ) in $b$ are contained in $p_{1}+p_{2}+p_{3}=$ $K_{1}$ or $q_{1}+q_{2}+q_{3}=K_{2}$. This is a straightforward computation (discuśsed below) which shows ad $b$ acts differently in $K_{1}$ and $K_{2}$ so that there can be no isomorphic irreducible ad $b$-submodules in $K_{1}$ and $K_{2}$.

Thus we see for $S \in J$ that $S K_{i} \subset K_{i}$ and $J_{0}$ consists of $r \times r$ symmetric matrices. For $J_{2}=J \mid K_{2}$ we note that

$$
\begin{aligned}
& U=\left[\begin{array}{llll}
0 & & & 0 \\
& b_{22} & & \\
& & b_{33} & \\
0 & & & b_{44}
\end{array}\right] \text { in } b, \text { and } \\
& X=\left[\begin{array}{lrrr}
0 & A_{12} & A_{13} & A_{14} \\
-A_{12}^{t} & 0 & 0 & 0 \\
-A_{13}^{t} & 0 & 0 & 0 \\
-A_{14}^{t} & 0 & 0 & 0
\end{array}\right] \text { in } K_{2}
\end{aligned}
$$


gives

$$
[U, X]=\left[\begin{array}{cccc}
0 & -A_{12} b_{22} & -A_{13} b_{33} & -A_{14} b_{44} \\
-b_{22} A_{12}^{t} & 0 & 0 & 0 \\
-b_{33} A_{13}^{t} & 0 & 0 & 0 \\
-b_{44} A_{14}^{t} & 0 & 0 & 0
\end{array}\right]
$$

so that ad $b$ acts differently on the $q$ 's and consequently the $q_{i}$ are homogeneous components (also see end of this example). Thus $s: q_{i} \rightarrow q_{i}$ and $s \mid K_{2}$ has matrix

$$
\left[\begin{array}{ccc}
S_{1} & 0 & 0 \\
0 & S_{2} & 0 \\
0 & 0 & S_{3}
\end{array}\right]
$$

To find the $S_{i}$ 's is essentially the same as in Example (1); thus we see $J_{2}=$ $J_{2}\left(q_{1}\right) \oplus J_{2}\left(q_{2}\right) \oplus J_{2}\left(q_{3}\right)$ where the $J_{2}\left(q_{i}\right)$ are isomorphic to the Jordan algebra of $r \times r$ sy.nmetric matrices.

To find $J_{1}=J \mid K_{1}$ we note for $U$ given as above and for

$$
Y=\left[\begin{array}{rrrr}
0 & 0 & 0 & 0 \\
0 & 0 & A_{23} & A_{24} \\
0 & -A_{23}^{t} & 0 & A_{34} \\
0 & -A_{24}^{t} & -A_{34}^{t} & 0
\end{array}\right]
$$

in $K_{1}=p_{1}+p_{2}+p_{3}$ that

$$
[U, Y]=\left[\begin{array}{cccc}
0 & 0 & 0 & 0 \\
& 0 & b_{22} A_{23}-A_{23} b_{33} & b_{22} A_{24}-A_{24} b_{44} \\
* & 0 & b_{33} A_{34}-A_{34} b_{44}
\end{array}\right]
$$

where $*$ is given by skew-symmetry. By making various choices for the $b_{i i}$ we see that the $p_{i}$ do not contain ad $b$-isomorphic irreducible submodules. Thus the $p_{i}$ are homogeneous components and are actually irreducible. Thus we see $S: p_{i} \rightarrow p_{i}$ so $S$ on $K_{1}$ has matrix

$$
\left[\begin{array}{ccc}
S_{1} & 0 & 0 \\
0 & S_{2} & 0 \\
0 & 0 & S_{3}
\end{array}\right]
$$


where the $S_{i}$ are symmetric on $p_{i}$. Therefore $S_{i}$ has a real characteristic root $\lambda_{i}$ on $p_{i}$ and $\left\{X \in p_{i}: S_{i} X=\lambda_{i} X\right\}$ is ad $b$-invariant. Thus by irreducibility, $s_{i}=$ $\lambda_{i} I$ on $p_{i}$; that is, $J_{1}$ is isomorphic to $R I_{1} \oplus R I_{2} \oplus R I_{3}$ where $I_{i}$ is the identity on $p_{i}$. Finally comparing the equations (1) and (2) above we see that we can choose the $b_{i i}$ so that ad $b$ acts differently on $K_{1}$ and $K_{2}$ so that the homogeneous components in $b$ are either in $K_{1}$ or $K_{2}$.

Example. (3) We now consider a case where the forms are not positive definite. Thus let $g$ be the $n \times n$ matrices of trace zero (that is, $A_{n-1}$-type) and let $b$ be the $k \times k$ matrices of trace zero imbedded as follows. Represent elements of $g$ by a sum of block matrices

$$
\left[\begin{array}{ll}
A_{11} & A_{12} \\
A_{21} & A_{22}
\end{array}\right]+R\left[\begin{array}{ll}
-I & 0 \\
0 & I
\end{array}\right]
$$

where trace $A_{22}=$ trace $A_{11}=0$. Let $b$ be represented by the $k \times k$ block of matrices

$$
\left[\begin{array}{ll}
0 & 0 \\
0 & b_{22}
\end{array}\right]
$$

where we use the notation $b_{22}=A_{22}$ and let

$$
V=\left[\begin{array}{cc}
-I & 0 \\
0 & I
\end{array}\right] .
$$

Again using $\operatorname{Kill}(P, Q)=\lambda_{\operatorname{tr}} P Q$, we see that $m=b^{\perp}$ consists of matrices of the form

$$
\left[\begin{array}{rr}
A_{11} & A_{12} \\
A_{21} & 0
\end{array}\right]+R V
$$

and $K_{0}$ is given by matrices

$$
\left[\begin{array}{ll}
A_{11} & 0 \\
0 & 0
\end{array}\right]+R V .
$$

Thus $K_{0}=c \oplus K_{0}^{\prime}$ where the center $c=R V$ and $K_{0}^{\prime}$ is isomorphic to the $r \times r$ matrices of trace zero where $n=r+k$.

Next $m=K_{0}+b$ and $b$ is given by matrices 


$$
\left[\begin{array}{lr}
0 & A_{12} \\
A_{21} & 0
\end{array}\right]
$$

and a computation shows $b=K_{2}$. Let $p_{1}$ be matrices of the form

$$
\left[\begin{array}{rrr}
0 & A_{12} \\
& & 0
\end{array}\right]
$$

and let $p_{2}$ be matrices of the form

$$
\left[\begin{array}{ll}
0 & 0 \\
A_{12} & 0
\end{array}\right]
$$

Then $(\operatorname{ad} b)\left(p_{i}\right)=p_{i}$ and $K_{2}=p_{1}+p_{2}$. The action of ad $b$ is given by

$$
\operatorname{ad} b\left[\begin{array}{lr}
0 & A_{12} \\
A_{21} & 0
\end{array}\right]=\left[\begin{array}{lr}
0 & -A_{12} b_{22} \\
b_{22} A_{21} & 0
\end{array}\right] \text {. }
$$

Let $A_{i}$ be a matrix of the form

$$
\left[\begin{array}{c}
0 \\
\alpha_{i 1} \cdots \alpha_{i k} \\
0
\end{array}\right]
$$

then matrices of form

(*)

$$
\left[\begin{array}{lr}
0 & A_{i} \\
0 & 0
\end{array}\right]
$$

yield isomorphic irreducible ad $b$-submodules of $p_{1}$. Similarly for $p_{2}$ using column matrices. However the $p_{1}$ and $p_{2}$ do not contain ad $b$-isomorphic irreducible submodules. Briefly, suppose $n_{1} \rightarrow n_{2}: X \rightarrow \bar{X}$ is such an isomorphism of irreducible submodules where

$$
X=\left[\begin{array}{ll}
0 & A \\
0 & 0
\end{array}\right] \text { and } \bar{X}=\left[\begin{array}{cc}
0 & 0 \\
\bar{A} & 0
\end{array}\right] .
$$

Then we obtain $b_{22} \bar{A}=-\overline{A b}_{22}$ and by suitably choosing the elements in $b_{22}$ (using 0's and 1's in the correct places) we obtain contradictions. Thus $p_{1}$ and $p_{2}$ are ad $b$-homogeneous components. 
Using the decomposition $m=K_{0}+p_{1}+p_{2}$ we see that $S \in J$ has matrix

$$
\left[\begin{array}{ccc}
s_{0} & 0 & 0 \\
0 & \bar{s}_{1} & 0 \\
0 & 0 & \bar{s}_{2}
\end{array}\right]
$$

so that $J_{0}$ is isomorphic to the $t \times t \quad B$-symmetric matrices where $t=r^{2}$ is the dimension of $K_{0}$.

Next on $b=p_{1}+p_{2}$, ad $b \mid b$ has matrix

$$
\left[\begin{array}{ll}
b_{1} & 0 \\
0 & b_{2}
\end{array}\right]
$$

where $b_{i}$ is the matrix of ad $b \mid p_{i}$; thus $[\operatorname{ad} b, S]=0$ gives $\left[b_{i}, \bar{s}_{i}\right]=0$. Now consider $\left[b_{1}, \bar{S}_{1}\right]=0$ on $p_{1}$; the case for $p_{2}$ is similar. Let $p_{1}=q_{1}+\cdots+q_{r}$ where the $q_{i}$ are the submodules given by $(*)$ above and $r=n-k$. By choosing a "natural" bases for each $q_{i}$ we see $b_{1}$ has matrix of the form

$$
\left[\begin{array}{llll}
H_{11} & & & 0 \\
& \cdot & \\
& & \cdot & \\
0 & & H_{r r}
\end{array}\right]
$$

where $H_{i i}=H_{j j}$ is $k \times k$ of trace zero. Now relative to this same basis, write

$$
\bar{S}_{1}=\left[\begin{array}{c}
s_{11} \cdot S_{r r} \\
s_{r 1} \cdot s_{r r}
\end{array}\right]
$$

then $\left[b_{1}, \bar{S}_{1}\right]=0$ gives $H_{i i} S_{i j}-S_{i j} H_{j j}=0$. But $H_{i i}=H_{j j}$ so that each $S_{i j}$ commutes with $k \times k$ matrices of trace zero. Since $S_{i j}$ commutes with the identity, we see $S_{i j}$ comnutes with all $k \times k$ matrices; thus $S_{i j}=\lambda_{i j} l$ so that $\bar{J}_{1}=J \mid p_{1}$ is isomorphic to the Jordan algebra of $r \times r$ matrices.

Similarly for $\bar{S}_{2} \in \bar{J}_{2}=J \mid p_{2}$ we obtain a matrix as follows. We note from $\operatorname{Kill}(P, Q)=\lambda \operatorname{tr} P Q$ that $B\left(p_{i}, p_{i}\right)=0$ and $B\left(p_{1}, p_{2}\right) \neq 0$. That is, $p_{1}$ and $p_{2}$ are related by the nondegenerate form $B$ and considered dual. Choosing a basis for $p_{1}$ from the decomposition $p_{1}=q_{1}+\cdots+q_{r}$ as above, we get a decomposition $p_{2}=q_{1}^{\prime}+\cdots+q_{,}^{\prime}$ such that $B\left(q_{i}, q_{j}^{\prime}\right)=0$ if $i \neq j$ and $q_{i}$ and $q_{i}^{\prime}$ are related by the nondegenerate form $B$ and considered dual. Choosing a natural basis for $q_{i}$ as in (*), the corresponding dual basis for $q_{i}^{\prime}$ is obtained by using the corresponding column matrices. 
Now let $\bar{S}_{2} \in \bar{J}_{2}$ have matrix $\left[\mu_{i j}\right]$ relative to $p_{2}=q_{1}^{\prime}+\cdots+q_{r}^{\prime}$ decomposition (as in the case for $p_{1}$ ). Then for $X_{i} \in q_{i}$ we have $\bar{S}_{1} X_{i}=\Sigma \lambda_{k i} X_{k}$ with $X_{k} \in q_{k}$ and for $X_{i}^{\prime} \in q_{i}^{\prime}$ we have $\bar{S}_{2} X_{i}^{\prime}=\Sigma \mu_{k i} X_{k}^{\prime}$ with $X_{k}^{\prime} \in q_{k}^{\prime}$. Using $S$ is $B$-symmetric and the orthogonal relations of the $q_{i}$ and $q_{j}^{\prime}$ we have by the usual computations that $\lambda_{j i}=\mu_{i j}$. This determines $J_{2}$.

Even though the algebras $(m, a, C)$ can be determined by finding $J$, these algebras are difficult to compute. In the next section we shall see how to obtain restrictions on $J$ by considering geometric formulas.

5. Some applications. We shall give some sample applications by considering various "geometrical" formulas which are functions of the connection by considering them as functions of elements of $M=J \cap G L(m)$. The formulas show these functions are analytic on the open set $M$ (in $J$ ) and we can compute their Taylor's series, find critical points, etc. Since we will always assume $I \in M$, we are considering a "perturbation" problem to see how formulas for a connection given by $S=I+t T \in M$ for $T \in J$ and $t$ near $0 \in R$ differ from formulas determined by a connection of the first kind.

First let $\nabla$ be a $G$-invariant pseudo-Riemannian connection on $G / H$ given by the algebra $(m, a, C)$ as in $\$ 1$. Thus we are assuming a pseudo-Riemannian connection of the first kind exists, given by $(m, 1 / 2 X Y, B)$, and we are comparing the connections via $C(X, Y)=B(S X, Y)$ which gives the elements $S \in M$. The connection formula for $\nabla$ given by $\alpha$ is

$$
2 a(X ; Y)=X Y+S^{-1}[X(S Y)-(S X) Y]
$$

for $X, Y \in m$ with $g=m+b$.

Let $M=J \cap G L(m)$ and let $L^{2}(m, m)$ be the space of bilinear maps on $m$, then define the function

$$
a: M \rightarrow L^{2}(m, m): S \rightarrow a(S)
$$

where $a(S)(X, Y)=a(X, Y)$ as given above. From the formula for $\alpha$, we see $a$ is analytic on $M$; and in particular $a$ is analytic at $I$, which gives the original connection of the first kind.

We now compute the Taylor's series for the function $a$ expanded about a point $P \in M$. Thus let $T \in J$ and $t \in R$ so that $P+t T \in M$ (and so that the following computations holds). From formulas,

$$
\begin{aligned}
2 a(P+t T)(X, Y)= & X Y+(P+t T)^{-1}[X \cdot(P+t T) Y-(P+t T) X \cdot Y] \\
= & X Y+P^{-1} A+t\left(P^{-1} B-U P^{-1} A\right) \\
& +\cdots+(-1)^{k-1} t^{k} U^{k-1}\left(P^{-1} B-U P^{-1} A\right)+\cdots
\end{aligned}
$$


where $U=P^{-1} T, A=X \cdot P Y-P X \cdot Y$, and $B=X \cdot T Y-T X \cdot Y$. From this we compute the higher derivatives $2 k !\left(D^{k} a(P)\right) T^{(k)}$ operating on $(X, Y)$ to be $(-1)^{k-1} U^{k-1}\left(P^{-1} B-U P^{-1} A\right)$. In particular if $T \in J$ is such that $(\operatorname{Da}(P))(T)=$ 0 , then $P^{-1} B-U P^{-1} A=0$ so that

$$
2 a(P+t T)(X, Y)=X Y+P^{-1} A=X Y+P^{-1}(X \cdot P Y-P X \cdot Y)
$$

which is the connection determined by $P \in M$.

Now suppose $P$ is a critical point of the function $a$; that is, $(D a(P)) T=0$ for all $T \in J$. Then from the above computations we see the Taylor's series is of the form

$$
\begin{aligned}
a(P+t T)(X, Y) & =(a(P)+(D a(P)) T+\cdots)(X, Y)=a(P)(X, Y) \\
& =1 / 2 X Y+1 / 2 P^{-1}(X \cdot P Y-P X \cdot Y)
\end{aligned}
$$

from (*). But by choosing $T=I \in J$ we see

$$
\begin{aligned}
0=2[D a(P) I](X, Y) & =P^{-1} B-U P^{-1} A, \text { in terms of } I \\
& =P^{-1}\left[X \cdot I Y-I X \cdot Y-P^{-1}(X \cdot P Y-P X \cdot Y)\right]
\end{aligned}
$$

which implies $X \cdot P Y-P X \cdot Y=0$; thus we see $a(P)(X, Y)=1 / 2 X Y$. That is a critical point of a gives a connection of the first kind.

Next we consider the perturbation of curvature. The curvature evaluated at $H \in G / H$ for the $G$-invariant connection $\nabla$ determined by $(m, a, C)$ is given by

$$
R(X, Y) Z=a(X, \alpha(Y, Z))-\alpha(Y, \alpha(X, Z))-\alpha(X Y, Z)-D(X, Y) Z
$$

(see [7] and note $\oint_{3}$ for $D(X, Y) Z$ ). Thus regarding $R$ as a function on $M$ we have an analytic map

$$
r: M \rightarrow L^{3}(m, m): S \rightarrow r(S)
$$

where $r(S)(X, Y, Z)=R(X, Y) Z$. Since we know $I \in J$, let $T \in J$ and $t \in R$ near enough 0 in $R$ so that $S=I+t T \in M$ and so that we can perform the following series computations.

From preceding computations we see

$$
\begin{aligned}
2 a(X, Y) & =2 a(I+t T)(X, Y) \\
& =X Y+(I+t T)^{-1}[X \cdot(I+t T) Y-(I+t T) X \cdot Y] \\
& =X Y+\sum_{k=1}^{\infty}(-1)^{k-1} t^{k} T^{k-1}(X \cdot T Y-T X \cdot Y)
\end{aligned}
$$


and using this in the above formula for $R(X, Y) Z$ we obtain the following for $r(S)(X, Y, Z)$ :

$$
\begin{aligned}
& 4 r(S)(X, Y, Z)=2 \alpha(X, 2 \alpha(Y, Z))-2 \alpha(Y, 2 \alpha(X, Z))-4 \alpha(X Y, Z)-4 D(X, Y) Z \\
& =X \cdot Y Z-Y \cdot X Z-2 X Y \cdot Z-4 D(X, Y) Z \\
& +\sum_{P=1}^{\infty}(-1)^{P-1} t^{P}\left\{X \cdot T^{P-1}(Y \cdot T Z-T Y \cdot Z)-Y \cdot T^{P-1}(X \cdot T Z-T X \cdot Z)\right. \\
& +T^{P-1}[X \cdot T(Y Z)-T X \cdot Y Z \\
& -Y \cdot T(X Z)+T Y \cdot X Z-2 X Y \cdot T Z+2 T(X Y) \cdot Z]\} \\
& +\sum_{k=1}^{\infty} \sum_{n=1}^{\infty}(-1)^{k+n} t^{k+n}\left\{T ^ { k - 1 } \left[X \cdot T^{n}(Y \cdot T Z-T Y \cdot Z)\right.\right. \\
& -Y \cdot T^{n}(X \cdot T Z-T X \cdot Z) \\
& -T X \cdot T^{n-1}(Y \cdot T Z \cdot-T Y \cdot Z) \\
& \left.\left.+T Y \cdot T^{n-1}(X \cdot T Z-T X \cdot Z)\right]\right\} .
\end{aligned}
$$

From this we can compute the derivatives $\left[D^{k} r(I) T^{(k)}\right](X, Y, Z)$; in particular $4\left[D_{r}(I) T\right](X, Y, Z)=X(Y \cdot T Z-T Y \cdot Z)-Y(X \cdot T Z-T X \cdot Z)+X \cdot T(Y Z)-T X \cdot Y Z$

$$
-Y \cdot T(X Z)+T Y \cdot X Z-2 X Y \cdot T Z+2 T(X Y) \cdot Z .
$$

If $I$ is a critical point of $r$, then we obtain a restriction on $J$ given by $\left[R_{1}(X, Y)\right.$, $T]=0$ for all $T \in J$ where $R_{1}(X, Y)=1 / 4[L(X), L(Y)]-1 / 2 L(X Y)-D(X, Y)$ is the curvature endomorphism for a connection of the first kind; recall $L(X): m \rightarrow m$ : $Y \rightarrow X Y$. To see this we have from $0=\left[D_{r}(I) T\right](X, Y, Z)$ as an operator on $Z$ that

$$
\begin{aligned}
0= & {[L(X), L(Y)] T-[L(X), L(T Y)]+[L(Y), L(T X)] } \\
& +L(X) T L(Y)-L(Y) T L(X)+2 L(T(X Y))-2 L(X Y) T .
\end{aligned}
$$

Taking the transpose of this equation, recalling $L(X)^{b}=-L(X)$ and $T^{b}=T$, we have

$$
\begin{aligned}
0= & -T[L(X), L(Y)]+[L(X), L(T Y)]-[L(Y), L(T X)] \\
& +L(Y) T L(X)-L(X) T L(Y)-2 L(T(X Y))+2 T L(X Y) .
\end{aligned}
$$


Adding these equations gives

$$
0=[[L(X), L(Y)], T]+2[T, L(X Y)]=4\left[R_{1}(X, Y), T\right] .
$$

Now let $H=\{e\}$ and let $G$ be semisimple, then $g=m$ and $L(X)=\operatorname{ad} X$. This gives $R_{1}(X, Y)=-1 / 4 \operatorname{ad}([X, Y])$ and $0=[\operatorname{ad}([X, Y]), T]$. But $g=[g, g]$ so we have $[\operatorname{ad} Z, T]=0$ for all $Z \in g$. This implies $[X, T Y]-[T X, Y]=0$ and therefore from the formula, $a(X, Y)=1 / 2[X, Y]$. This proves the following.

Proposition 5. Let $G$ be a semisimple connected Lie group with Lie algebra g. Let $r: M \rightarrow L^{3}(g, g)$ where $r(S)(X, Y, Z)=R(X, Y) Z$ is the curvature determined by $(g, a, C)$. If $I \in M$ is a critical point of $r$, then the connection determined by $(g, a, C)$ is of the first kind; that is $a(X, Y)=1 / 2[X, Y]$.

Examples indicate similar results hold for reductive pairs $(g, b)$ with $g$ and $b$ semisimple where $g=m+b$ with $m=b^{\perp}$ and $m m \not \equiv$; that is, $(g, b)$ is not a symmetric pair.

\section{REFERENCES}

1. E. B. Dynkin, Semisimple subalgebras of semisimple Lie algebras, Mat. Sb. 30 (72) (1952), 349-462; English transl., Amer. Math. Soc. Transl. (2) 6 (1957), 111-244. MR 13, 904.

2. N. Jacobson, Structure of rings, Amer. Math. Soc. Colloq. Publ., vol. 37, Amer. Math. Soc., Providence, R. I., 1956. MR 18, 373.

3. - Lie algebras, Interscience Tracts in Pure and Appl. Math., no. 10, Interscience, New York, 1962. MR $26 \# 1345$.

4. B. Kostant, On holonomy and homogeneous spaces, Nagoya Math. J. 12 (1957), 31-54. MR $21 \# 6003$.

5. O. Loos, Symmetric spaces. II, Benjamin, New York, 1969. MR 39 \#365b.

6. K. McCrimmon, On Herstein's theorem relating Jordan and associative algebras, J. Algebra 13 (1969), 382-392. MR $40 \# 2721$.

7. K. Nomizu, Invariant affine connections on homogeneous spaces, Amer. Math. J. 76 (1954), 33-65. MR 15, 468.

8. A. Sagle, On simple extended Lie algebras over fields of characteristic zero, Pacific J. Math. 15 (1965), 621-648. MR 32 \#7612.

9. - Some homogeneous Einstein manifolds, Nagoy a Math. J. 39 (1970), 81106. MR $42 \# 6748$.

10. A. Sagle and D. Winter, On homogeneous spaces and reductive subalgebras of simple Lie algebras, Trans. Amer. Math. Soc. 128 (1967), 142-147. MR 37 \#2910.

11. A. Sagle and J. Schumi, Multiplications on homogeneous spaces, nonassociative algebras and connections, Pacific J. Math. (to appear).

DEPARTMENT OF MATHEMATICS, UNIVERSITY OF MINNESOTA, MINNEAPOLIS, MINNESOTA 55455

DEPARTMENT OF MATHEMATICS, HILO COLLEGE, UNIVERSITY OF HAWAII, HILO, HAWAII 96720 (Current address) 